\title{
Direct Conversion of Standard Sea Water Electrolyte to Electrical Energy
}

\author{
Sugandar Sumawiganda $\mathrm{a}^{\mathrm{a}, \mathrm{b}^{*}}$ \\ ${ }^{a}$ CIETRIG-MEDCO_Energy,Inc. \\ ${ }^{b} I T B$ Gedung PLN, Jln Ganesha 10 Bandung, Indonesia
}

\begin{abstract}
This paper explains the techno-economical feasibility of very efficient direct energy conversion of standard seawater electrolytes to electrical energy based on solid state power electronics. It can be shown by applying free Gibbs energy concept and chemical REDOX reaction that the ions are in higher entropy than their associated solid crystal's form, and therefore they have higher potential energy. The key problem is obviously to separate these free ions in separate subspaces according to its charge, since this separation will create electrical potential difference between them. The ions are essentially electrons carrier and therefore despites they are moving with random velocity magnitude and direction, the external observer will observe an increasing electric charge due to free ions influx. This separation and migration can be performed by a rather strong electric field created by a special varnish insulated wire grid, VIWG. It will be shown that the energy expenditure for the liberation of ions from hydrates structure containment and migrate them to separate subspaces is similar to energy activation for the oxidation exothermal process, which requires only marginal external energy. Thus the energy gain is $250 \mathrm{kJoule} / \mathrm{kg}$ standard seawater, which is only $0.55 \%$ of the fuel value of $1 \mathrm{~kg}$ Texas' crude oil, U.S.A. Unlike thermal energy conversion, this system may has $95 \%$ efficiency, since all rotating machines are eliminated. With this efficency, the "priceless" seawater feedstock leads to the electric energy tariff which can be as low as $30 \%$ of the current one. In addition, this system provides with a number of significant comparative and competitive advantages compare to any known energy generation technology today, a.o.: zero pollution process, significant by products (fresh water, hydrogen gas, pure solid crystal atoms precipates) renewable since the availabilty of seawater feedstock is unlimited, scalable, modular, small foot-print, co-generation of Sodium/Chlorine fuel cell, and table salt ( $\mathrm{NaCl})$, and conventioanl hydrogen/oxygen fuel cell. It can be modified as power house for any kind of ship, as small as traditional fishing vessel to ocean liner.
\end{abstract}

Keywords: Gibbs free energy, REDOX, hydrates, zero polution, fuel cell, scalable, modular

\section{Introduction}

This paper explains the techno-economical feasibility of very efficient direct energy conversion of standard seawater electrolytes to electrical energy based on solid state power electronics. It can be shown by applying free Gibbs free energy concept and chemical REDOX reaction (Brown et al. 1994; Chang 2007) that the ions are in higher entropy than their associated solid crystal's form, and therefore they have higher potential energy.

The key problem is obviously to separate these free ions in separate subspaces according to its charge, since this separation will create electrical potential difference between them. The ions are essentially electrons carrier and therefore despites they are moving with random velocity magnitude and direction, the external observer will observe an increasing electric charge due to free ions influx. This separation and migration can be performed by a rather strong electric field created by a special varnish insulated wire grid, VIWG (Sumawiganda 2012a).

It will be shown that the energy expenditure for the liberation of ions from hydrates structure containment and migrate them to separate subspaces is similar to energy activation (Fishbone et al. 2005; Brown et al.

* Corresponding Author:

Email: suci2308@yahoo.co.id 
1994) for the oxidation exothermal process, which requires only marginal external energy (Sumawiganda 2012a). Thus the energy gain is $250 \mathrm{kJoule} / \mathrm{kg}$ standard seawater, which is only $0.55 \%$ of the fuel value of $1 \mathrm{~kg}$ Texas' crude oil, U.S.A. (Brown et al. 1994). Unlike thermal energy conversion, this system may has $95 \%$ efficiency, since all rotating machines are eliminated.

With this efficency, the "priceless" seawater feedstock leads to the electric energy tariff which can be as low as $30 \%$ of the current one. In addition, this system provides with a number of significant comparative and competitive advantages compare to any known energy generation technology today, a.o. : zero pollution process, significant byproducts (fresh water, hydrogen gas, pure crystal atoms) renewable since the availabilty of seawater feedstock is unlimited, scalable, modular, small foot-print, co-generation Sodium/Chlorine fuel cell, and table salt $(\mathrm{NaCl})$ (Sumawiganda 2012b). It can be modified as power house for any kind of ship, as small as traditional fishing vessel to ocean liner.

\section{Material and Method}

\subsection{General concepts}

This comprehensive introduction will give a big picture of this new renewable energy generation technology based on the chemical energy content of seawater electrolytes. Analogies and well known similarity phenomena are explored to give enforcements of the associated basic concept which can be stated as (1) that the isolated artificial electric field can perform ulimited works without additional external energy. (2) that the free separated ions according to their charge have an electrical potential between them which can be utilized for electric energy generation. And (3) since direct conversion is non-thermal, its efficiency can be as high as above 95\% (Sumawiganda 2012b; Sumawiganda 2012c).

This new ground breaking technology converts directly the chemical potential energy content of seawater electrolyte in forms of ions hydrates to electrical energy without rotating machine, first by destroying the hydrates structure using a rather strong electric field, liberating both the surrounding water molecules and ions (Sumawiganda 2012a). The electric field creates a electric force $\mathbf{F}_{\mathbf{e}}$ that works on free polar water molecules and free ions. This same electric force activates a dipole moment and rotates the water molecules such that they allign themselves with local electric field lines. With this configuration the dipole moment becomes zero.

This process is depicted as water molecules polarization with very short polarization time, perhaps in the order picosecond. In the latter, the electric force performs displacement of the charge centroid of an ion in the directions of the local electric field line. In this temporal condition the free water molecules as well as the ions are in stressed condition similar to a stressed spring in mass-spring of a mechanical system (Kasap 2006). Thus when the action force dissapears, energy is releasead by the relaxing spring. Similarly when the stressed free water molecules and free ions are leaving the EFCV (Electric Field Control Volume) the electric field regain its stored energy back.

Obviously, there is no external energy expenditure of electric field used for polarization of water molecules and displacement of charge centroids effect of the free ions, provided that number of water molecules and ions entering the EFCV is exactly equal to the number leaving from it. Any discrepancy will be recorded by the external energy source as instantaneous electric current leaving or entering it.

\subsection{Intrinsic characteristic of the conversion technology}

This conversion technology has several intrinsic characteristic features to be familiar with in order to comprehend and appreciate the remarkable behaviour, which will presented in a moment.

\subsubsection{Elastic collision between free ions and free water molecules}

Another phenomena that needs to be considered is the collision effect with free water molecules imparting the so called impuls I and impuls force $\mathrm{F}_{\mathrm{im}}$, (Fisbone et al. 2005). This impuls and its associated impulse force work in very short period, in the order of nano-second. Afterward it becomes zero.

Recall that the water molecules are continuously in the state of random motion, both in term of its magnitudes and directions due to thermal energy agitation. This random motion is known as Brownian motion (Fishbone et al. 2005). The direction of the motion is independent of the velocity vector magnitude. The velocity magnitude is described 
probabilistically by Maxwell-Boltzmann continuous distribution function with temperature as parameter (Fishbone et al. 2005). The distribution curve shows a single convex crest depicted as a bell shaped form, which is almost symmetrical for low temperature similar to Gaussian probability curve, but skews to the right for higher temperatures. The values ranges from zero to its maximum probability.

Since the direction is in space, then the motion have an infinite degree of freedom. This thermal agitation is the sole driving force for diffusion phenomena of any particulate matter less than 1 micron in size in gas as well as in liquid phase and is not affected by electric, magnetic or gravitational field.

The most important point is that the the impuls force works only in a very short period of the order of nano second and its magnitude is very small but greater than gravitational force. Because of probabilistic nature we use the rms (root mean square) impuls force which is greater then the gravitational force. This explains why a suspended pigment solution is diffused in water and stay forever there.

The conclussion is that gravitational force and collision force are almost not significant compare to electric field force. Therefore we can neglect them at the onset, and we treat the motion of the marked ions as if there were no collisions! This similar to CRT (cathode ray tube) or X-ray tube where the tube is evacuated from air to prevent collisions with air molecules.

The distinct difference is that the electrons motions study is focused outside of electric field created between cathode and anode but still within the tube space, while in our case, our study of the motion of the marked Sodium ion is the interior of the electric field sub space. Detailed theory about this phenomena are given in refference (Sumawiganda 2012a). Figure 1 depicts the forces acting on a cation and anion (not shown) under the influence of electric field.

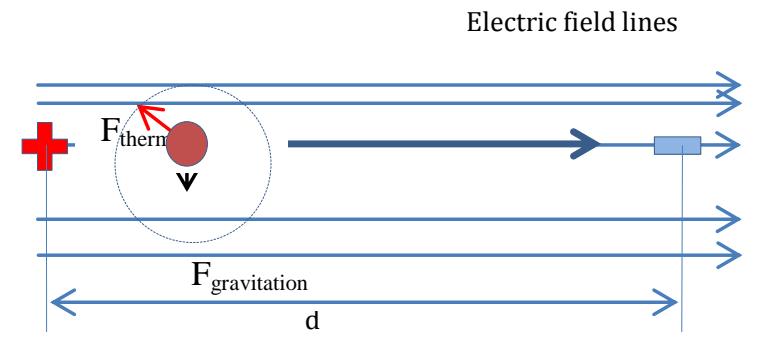

Fig. 1 Forces on a cation under influence of strong electric field. The work perfomed by the electric field is practically $\mathrm{W}=\mathrm{d}\left(\mathrm{F}_{\mathrm{e}, \mathrm{Na}+}+\mathrm{F}_{\mathrm{e}, \mathrm{Cl}}-\right)$ if $\mathrm{F}_{\mathrm{e}}$ million times the $\left|\mathrm{F}_{\text {thermal }}\right| \approx\left|\mathrm{F}_{\text {gravitation }}\right|$. Note that $\mathrm{F}_{\mathrm{e}}$ is underscaled in the figure and the anion is not shown, but its motion is directed opposite to electric field line. The circle is the projection of a sphere onto page, which is the locus of the direction and force magnitude due to thermal agitation, which disappear just after collision. Note that once the electric field is established, it can perform unlimited number of ions migration out of electric field without additional external energy, which is analogous to gravitational field even if the power supply is cut off. Forexample, if the artifical electric field is created by a flat plate capacitor where its capacitance is greater than 10 ? F, and $\mathrm{d}=2 \mathrm{~cm}$, then the capacitor can be isolated by disconnecting its power supply by a simple mechanical switch, while retaining its electric field. Note, we use a very small horizontal scale to accomodate the electrical field force $\mathrm{F}_{\mathrm{e}}$.

\subsubsection{Discrete time unit: The migration cycle (mcyc)}

Our system is physically a continous system, which means that any variable can be measured at any instant of time, forexample our system is steady fed by standard seawater then we expressed that the rate of input is $\mathrm{Q} / \mathrm{unit}$-time. The accumulated volume is then $\mathrm{Q}$ $X$ unit-time. In future discussion we will drop the adjective standard since we will assume that we work only with standard seawater, unless otherwise stated. In SI system the unit time is second. However, if we measure the varible at an instant of time, the measuring device record the instantaneous value of the variable, not its rate.

For numerical model we will introduce a discrete time unit of 2.5 ? 3 , with a definition that all variables pertaining to the continuous process will be expressed as accumulated (integrated) with this time unit (Sumawiganda 2012a, Sumawiganda 2012b, Sumawiganda 2012c). For convenience we will depict this discrete time unit as ions migration cycle (mcyc), which is the traversing time of an ion initially located at the maximum potential energy that moves along the energy field lines, as shown in Figure 1. We will adopt mcyc as standard time unit, derived from traversing 
time of Chlorine anion, $\mathrm{Cl}^{-}(\mathrm{f})$ where $\mathrm{f}$ in the bracket signifies a free chlorine anion.

Clearly, there are many different values of mcycs equal to the number of ions populations. There are two reasons why the Chlorine anion was selected as standard for mcyc instead of its pair, Sodium cation: (1) It is because the Chlorine anion is heavier than Sodium cation, therefore it moves slower, and (2) the molarity concentration $(0.5 \mathrm{M})$ of these ions is dominant $(85 \%$ by weight) compare to others constituents, which are usually heavier. Therefore we can assume that seawater is populated by only Sodium and Chlorine hydrates, which leads to molarity ratio of ions pair to water (solvent) about 1 to 111 . With this assumption only $85 \%$ desalination is achieved, which means that the desalinated seawater contains $0.075 \mathrm{M}$ impurities. This means that a batch process may produce perfect desalination if for a rather longer time. Therefore for continuous process we need a rather deep electric field control volume.

Obviously, when these free ions influx enter the separate zero electric field sub-spaces, their kinetic energy are damped by millions collisions with water molecules to lower velocities appropiate for the formation of new hydrates which moves (Brownian) randomly driven by thermal energy. Externally these hydrates are charge structure since the protuding poles carry electric charge as shown shematically in Figure 2. However due the thick shielding effect of water molecules the electrostatic attraction between them can be neglected.

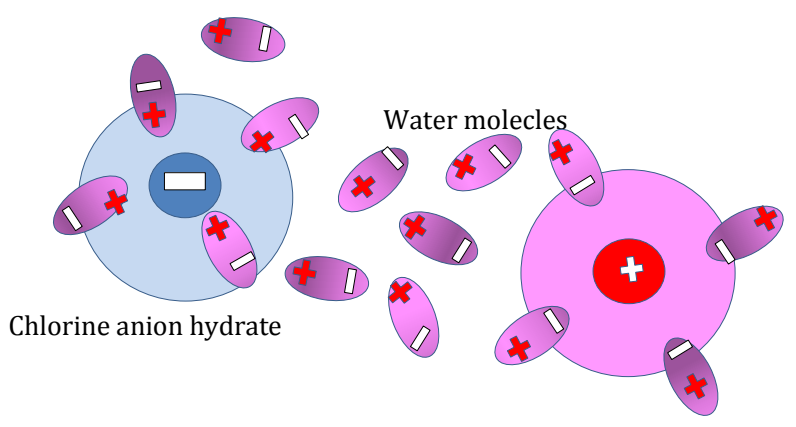

Fig. 2 Thermal dispersion will force the hydrates to be diffused uniformly, since the attrative electrostatic force is less then dispersion force. This is true if the TDS (total dissolved solids is less than 35 gram/liter). Greater than this value the attractive electro-static force may overcome the dispersive force, and the solution starts to precipates, but then other free water molecules will immediately start to form new hydrates. The net result is a dynamic equilibrium between precipitations and dissolution.

Recall that these hydrates are in constant random motion and therefore they experience unlimited number of collisions with free water molecules. Thus we can immagine that the hydrates are temporally destructed and rebuild again a million times in a very short period.

If we immerse a rod graphites then energetic collissions with the graphite surface will eventually occurs. Experiment has shown (Fishbone et al. 2005) that on average only $1 \%$ of this thermal collisions are greater than the activation energy threshold that leads to electrons exchange between ions and graphite's surface, donating or accepting electrons, provided that the graphites are isolated, i.e not connected to external conductor.

Obviously an electric potential exists between these terminals. Now, when the graphite's terminals are connected with external conductor this potential difference generates electric current, or eletrons flows from the anode to cathode. Our experiment in year 2012 have shown that this development has occured in a few second.

There are two possible development scenarios. First, if the graphites terminals are isolated, then electrons exchange occurs due to energetic collisions of ions and graphite's external surface, until all free electrons of the graphite's bar are donated to Sodium cations; and in other compartment, all excessive electrons are transfered from ions to graphite's terminal. The former process is exothermic oxidation releasing energy in form of heat, while the latter is endothermic reduction process. In the former energy is released in form of heat, and in the latter energy is aborbed from environment causing temperature's drop. Eventually the surface of the graphite becomes saturated, i.e it cannot perform no longer support electrons exchange with the ions. If the ions influx is maintained, then concurrently the free water molecules are expelled from the compartment. With this condition, the graphites terminal becomes a simple electrodes, that transmits high electric potentials and current created by "ions cloud" space capacitor when the two terminals approach each other as shown in Figure 3. This phenomena is similar to atmospheric ions cloud space capacitor which creates lighting, and thunder. 


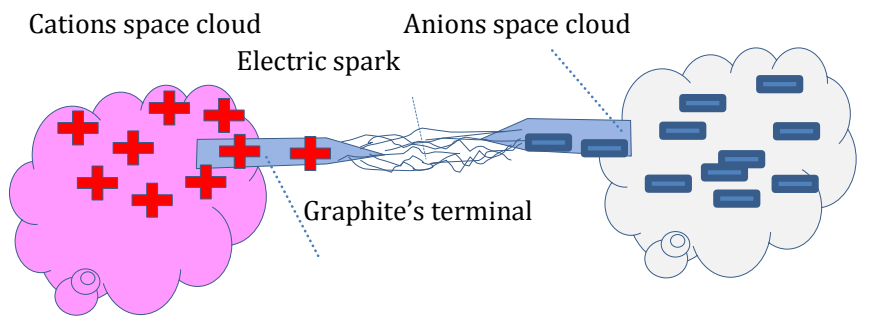

Fig. 3 Free $\mathrm{Na}^{+}$"cloud" and free $\mathrm{Cl}^{-}$form a space capacitor may develope high electric potential and current, that jump through a gap between graphites charge collector. In reality the space cloud is contained by finite ions compartment.

Second, if the graphite terminal are connected at the onset, the potential difference forces spontaneously the electrons flows from anode to cathode's terminal. In this case the electrical potential is governed by REDOX reaction which leads to 5.1 volt, independent of surface area of the rods. This is equal to emf (electric motive force) of a Galvanic cell. However, since the ions influx is maintained, it is more analogous to fuel cell. Note that a huge potential attributed to space capacitor effect did not occur, if and if the collectror graphite's rod is permanently connected. This implies that the graphite's rod and external conductor act as current moderator similar to graphite's moderator in nuclear energy plant.

It should be noted that EFCV created by VIWG capacitor acts as electric shield that prevent the penetration of electric field radiated from the rod collector. However, if we want to demonstrate the huge potential different of the ions space capacitor the permanent graphite's rod should be in off position, and another large number of graphite plate collector should be used to accomodate electrons avalance. In this case the REDOX reaction is overuled by capacitor's physics. Detailed discourses of this phenomena is given in the refferece (Chang 2007; Kasap 2006).

The remaining query is why the collisions in seawater electrolytes with ions are elastic. Experiments has shown that energetic collision neither produce light, nor sound, or heat. To investigate the latter we need a very sensitive thermometer and the experiment should be perform within a bomb-calorimeter, which is a very complex arrangement.

The physical implication of the above argument is that the collision should be elastic, where the energy is conserved. Consequently momentum and energy are conserved. It involves only the outer valence electron where the orbit changes temporary, without change in electron-spin and no displacement of internal electrons. In fact this is the only valid collision mode.

However, the collisions of ions with graphite's bar is not conservative, which means it involves energy loss. To minimize the energy loss, the collision energy should be just above the activitation energy level. At this point the author assumes that the activation energy level consumes $5 \%$ of the energy generated by autoelectrolysis.

\subsubsection{Zero energy requirement for ions separation and migration}

Next we consider the work performed by the electric field to migrate a single free ion out the EFCV. This work depends on the initial coordinates of the free ion, its type and charge. Let us consider a single Sodium cation, and the coordinates is initially at the electric field extremity where its potential energy is maximum. By Newton second law, the electric field force that works on this marked Sodium cation induces an accelerated motion a hundred million times greater than earth gravitational acceleration $g$. Thus we see that gravitational and collison effect can be neglected at the onset. The physical implication is that the gravitational and the collision impact force is not felt by our marked ion, thus they can be considered as non-existence. Kinematically, this means that the marked ion can be immagined as traversing in non-gravitational and frictionless water medium. This means that the marked ion moves exactly along the field energy lines and the escape velocity, i.e the velocity of our marked ion when leaving the electric field can be predicted exactly. It is equal to $15850 \mathrm{~m} / \mathrm{s}$ for Sodium cation over $d=2 \mathrm{~cm}$ gap, and with traversing time of 2.5 ? $s$.

The positive electric field work performes $W=F_{e} d / 100$ $=\operatorname{mad} / 100$ Joule, where $m$ is the mass of the Sodium cation and $a$ its constant acceleration. We designate the positive field work as downhill work, analogous to a gravitational field works on a falling rock over the cliff, or roller coaster vehicle downward movement along its track.

Unfortunately, there is a significant difference between gravitational field and electrical field work. The rock mass is part of the earth mass. External energy is required to lift the rock to an elevated position, thereby increasing its potential energy. Unlike a clump of rock which is part of the earth mass, the ions are not part of the electric field and they are created by 
dislodging ions from its solid crystal rock by the intrinsic electrostatic dipolar attractive force of polar water molecules. Thus the work has been done by natural forces and is free for us, which is a unique feature of all conventional renewable enegry like wind and tide energy.

When this rock falls due to gravitational downward pull, the potential energy is converted to kinetic energy, and if the system is assumed conservative the kinetic energy is exactly equal to potential energy.

Let us consider the electric field work to migrate ions from EFCV. The capacitance of the VIWG should be at least 10 ?Farad in order to maintain its electric field when the power source is cut off by a mechanical switch. If we used an electronic switch, lower capacitance value may be still maintained. Thus when the powers source is cut off, the field strength is retained and can still perform positive field work for ions migration. We say that the capacitor is isolated when the umbilical cord is cut off. Now since it is cut off from the power source, no external work is required and the similarity with the gravitational field is exact.

Another alternative is by considering the field which is still connected to the power source. The pure water dielectric $K_{\text {water }}=K_{w}=80 . K_{\text {seawater }}=K_{s w}$ can be expressed as $K_{s w}=K_{w}+\Delta$. Therefore the capacitance formula can be written as $C_{s w}=\left(K_{w}+\Delta\right) ?_{0} A_{0} / d E-100$ Farad. Obviously $C_{w}$ $=K_{w}$ ? ${ }_{0} A_{0} / d E-100$ Farad. Thus when the seawater penetrates the EFCV, the dielectric constant increases with an amount $\Delta$ due to the presence of "ions impurities". The power source responds with an electric current that enters the positive leg of the capacitor from the negative leg. When all ions are leaving the EFCV, the discrete model stipulated that the EFCV is only populated by fresh water, and hence $\Delta=0$ and the field regain its original energy.

At steady flow when the number of ions entering the control volumes $\equiv$ to the number of ions leaving the EFCV then the energy loss is equal to energy gain, and we will observed no electric current is leaving/enetering the power source. Thus if the hydraulic flow is perfectly steady and the ions are perfectly dispersed, the power supply will not send any current leaving or entering the capacitor. This fact implies zero work of the power supply surce for performing steady flow migration of the ions by electric field if the power supply is connected. This seems to be against the energy conservation law, however remember is the flow is perfectly steady the loss and gain of energy occurs concurrently, hence the sum is zero.

Next suppose the power supply is cut off and the the capacitance is sufficiently large, the capacitor will retain its charge, i.e it maintains its capacitance $C_{S w}$. Now suppose the hydraulic flow is perfectly steady, but the number of ions changes to new concentration $Q^{\prime}<Q_{\max }$, then the isolated capacitor responds is $V^{\prime}<V_{\max }$, so that its capacitance does not change.

An alternate view that the artificial electric field created by VIWG does not need a power supply source for ions migration, is the fact that ions (as electric current carrier) and capacitor do not form a closed circuit as forexample in conventional electrolysis, CRT, old TV tube and X-ray tube. Yet we know that the power supply does respons to the change of dielectric in form of electric current flow between the two electrode plates, for example if pure water dielectric changes in mcyc period, positive electric current will enter the positive plate and an exact amount of negative current will leave the negative plate (because of electrons conservation law) (Kasap 2006). Now in steady flow the mcyc $\equiv 0$, and we can not detect this changes.

Thus essentially the VIWG capacitor is an ideal transformer or converter, where the potential energy of an ion is converted to its equivalent kinetic energy without loss. This is analogous to an ideal electric AC power transformer, where the the voltage is converted to another voltage without energy loss (Rizzoni 2013; Hart 2011; Bogard Jr. et al. 2004).

The distance between collision is depicted as meanfree-path ?, which is a local and time dependent variable ? $=$ ? $(\mathrm{x}, \mathrm{y}, \mathrm{z}, \mathrm{t})$ (Fishbone et al. 2005). To simplify the numerical model we define $e m f_{r m s}=$ constant, which is derived from thermal velocity rms. We designate this model as random walk model. Despite this bold assumption, the end result is anticipated to be the same compare to more correct but very time intensive simulation model. It should be reminded that no one has attempt to apply correct simulation model. So at least at this time we adopt this statement at face value. There are many instances that the numerical simulation model does not conform to physical model, but the end result is correct. In this case the numerical model should be treated as black box. In some numerical simulation model produces non-physical variables which is related to discretization of a continuous variables to discrete one. With this behaviour, experiments becomes absolutely neccessary 
to interpret nonphysical result that creeps into the simulation model.

The ? $_{\mathrm{rms}}$ is a very short distance about ten times the diameter of water molecule. Despite a very small distance, the collision velocity, i.e. the collision velocity over this distance of a marked Sodium cation is relatively large of the order of $1500 \mathrm{~m} / \mathrm{s}$. This the minimum escape velocity of a marked ion initially very close to the exit point.

\subsubsection{Electric field ions separation, electric curtain}

In a well known reverse osmosis (RO) technology, the ions are expelled from any controlled volume space (CVS) by applying pressure slightly greater than osmotic pressure where the ions are forced to pass through a semi impermeable membrane. Thus all types of ions are froced to exite from this CVS by applied pressure and populate the same space outside the semiimpermeable membrane, but only more dense. In our case the ions are leaving the EFCV by electric field force, while the pressure does not change. Furthermore unlike RO the ions are separated according to its charge. They are dispersed uniformly in finite separate compartment and create electrical potential difference. Furthermore, once they are outside the EFCV, back diffusion is limited to electric double layer formation that will be explained in a moment. Thus the VIWG act as an electric curtain where the ions are verboten to cross.

\subsubsection{Electric double layer}

When the passing ions are to close to the surface of the VIWG, then the Coulomb's electro-static-attraction starts to be expressive which attract the passing ions to itself. It can be shown that this Coulomb force is relatively very large compare to the electric field force $\mathbf{F}_{\mathrm{e}}$. Then the inevitable fate of this ion is to collide with the wire, and stay there forming an electric double layer (EDL). Essentially this EDL consist of a fractal like surface in space where the separation charge distance is about $1 / 100 \mathrm{~mm}$. The electric shield effect is similar to Faraday cage (Figure 4) where the electric field is forced to curve around it. The field intensity changes to half the original value assuming that its effect extent to $d=2 \mathrm{~cm}$. This is because half of the field lines are arrested by EDL. To overule this effect, the impressed voltage should be increased to twice of its original value. This adjustment should be perform automatically to prevent human error if manually controlled, in particular for large plant, such as 1000 MWatt plant. This engineering aspect can be solved by modern state of the art electronic power control.

\subsubsection{Event horizon layer $(E H L)$}

An event horizon layer is the top layer of the EFCV with a thickness depth of $d=5 \times 10^{-6} \mathrm{~m}$. This is the site where the free ions are migrating within migration time of 2.5 ? $\mathrm{s}$. With a feedstock of $Q_{s w}=1$ liter $/ \mathrm{s}$ and entry area of $100 \mathrm{~cm}^{2}$, the seawater penetration velocity is 2 $\mathrm{m} / \mathrm{s}$ the penetration volume is $5.5 \times 10^{-5}$ liter $=5.5 \times 10^{-2}$ $\mathrm{cm}^{3}$. The number of ions is $3 E+23 \times 5.5 E-5=16.5 E+18=$ $1.65 E+19$ pairs of $\mathrm{Na}^{+} / \mathrm{Cl}^{-}$per $2.5 E-6$ seconds. Thus the model stipulates that the EVL is emptied every 2.5 ? $s$, and replaced by a new ions originating from seawater feedstock. The number of ions emitted through a slit of 5 micron depth and $10 \mathrm{~cm}$ width, which is similar to a continuous burst from a multi barrel ions cannon (MMIC) analogous to electrons gun of a CRT.
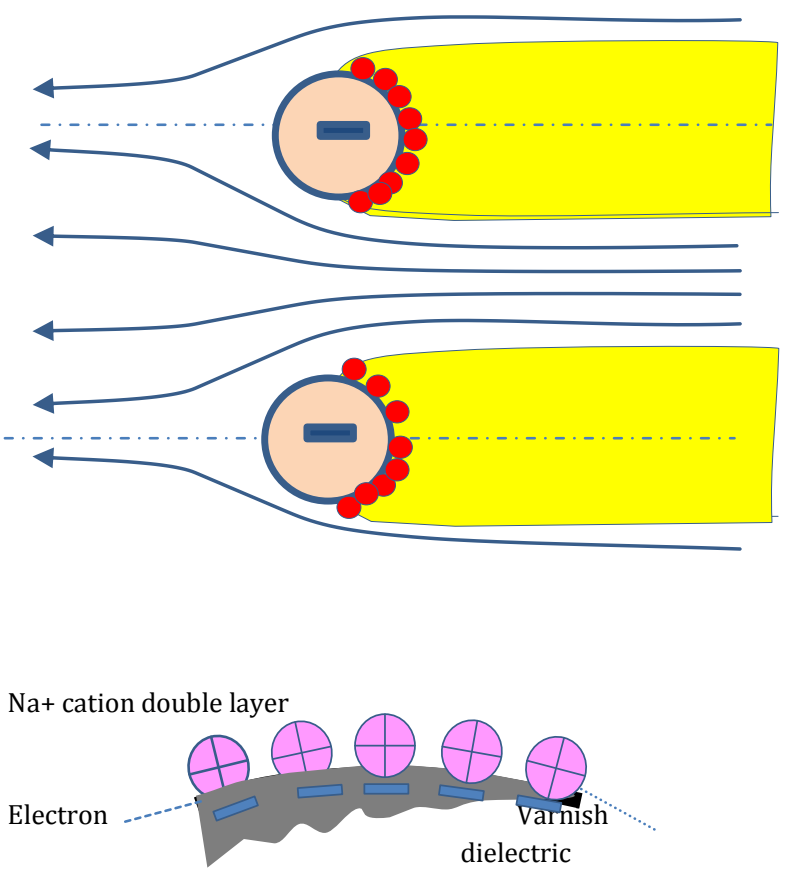

Fig. 4 Cross section of (-) VIWG covers by $\mathrm{Na}+$ cation double layer plak. Note that only one layer effective plak, which act as Faraday cage. Thus inside the yellow color the electric field is zero. However, if the power supply is on, the potential difference will be maintained by compensation electric current from it. 


\subsubsection{Load matching problem}

The ions influx into the cation and anion compartment should be immediately neutralized. Continuous neutralization of ions influx can be achieved if the energetic ions influx collides with the immersed graphite's bar where the collision energy is utilized for electrons exchange between graphites and ions. However, since the surface area of the graphite's surface is limited, this process will be soon terminated, because very soon the saturation condition will be attained. By connecting the graphite's rod terminals with external conductor, the existing electric potential difference will drive the electrons from the anion to cation rod. Then the graphite's terminal behaves as a pure electrons collector. Thus a matching problem is solved.

Nevertheless another problem emerges. The neutralized ions are increasingly accumulated in both compartments which eventually suffocates the system. To prevent this to occur, the precipated neutral atoms in the cations compartment and the dissolved carbonates, phosphates, nitrates, sulphates in the anions compartment should be removed continuously, such that over accumulation is prevented. This is of course an engineering design problems that should be properly solved.

When the graphite's terminal is connected by external conductor, a stable electric current will be established. This phenomena is depicted as autoelectrolysis, which is similar to fuel cell, where the seawater feedstock is supplied continuously. Note that ions influx is expressed as $Q_{i f}=$ ions influx $/$ mcyc (2.5 ?s).

In our study case the $Q_{i f}=0.5 \mathrm{M}$, i.e ions influx per second. $Q_{i f}$ is non-negotiable, which means that the ions influx should be immediately neutralized by the auto-electrolysis process. Lower than the stipulated rate will cause accumulation. Since $Q_{i f}$ oscillates over its rms average, the graphite's interceptor should be large enough to accomodate this inherent fluctuation.

\subsubsection{Passive power control, auto-electrolysis}

The above discussion leads to the passive control of generated electrical potential of 5.1 volt by forcing REDOX reaction (as oxydation of Sodium ions) to occur at the surface of the graphite's rod in the cation compartment. The graphite's rod is depicted as cathode. On the opposite side, the REDOX reaction occurs as reduction of Chlorine anion in which energy is directly absorbed from the collision of the Chlorine's anion with grahite's surface to overcome the electric energy barrier.

Thus the excess electron of a Chlorine anions jump to the graphite's surface. When this occurs the graphite becomes an anode and transmits electrons flow to the cathode. Obviously the $5.1 \mathrm{ev}$ energy released at the cathode surface is intact, i.e not substracted for the reduction process of Chlorine anions where $3.8 \mathrm{ev}$ is absorbed. Recall that $1.3 \mathrm{ev}$ is produced due to the compounding formation of $\mathrm{NaCl}$ molecule. This is essentially an auto-electrolysis which contrary to common electrolysis, electrical energy is generate

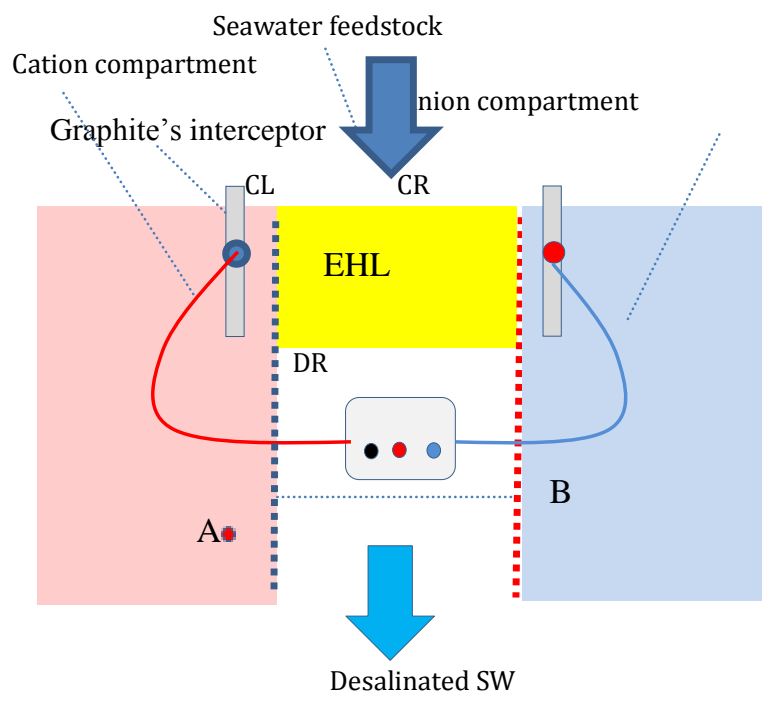

Fig. 5 Part of the side View of EHL (Event Horizon Layer, collored yellow) and Twin CL-DL and CR-DR (Multi barrel ions cannon). The energetic free ions strike the graphite's interceptor where electrons exchange occur on the collision point between ions and graphite surface. Energy released by the oxydation of a single $\mathrm{Na}+$ is $5.1 \mathrm{ev}$ (electron volt) and energy absorbed by reduction of $\mathrm{Cl}$ - is $3.8 \mathrm{ev}$. The graphite terminals are permanently connected and moderate the voltage to fuel-cell 5.1 volt. $\mathrm{AB}$ is the instant seawater penetration, but notice all ions have been EHL and below the EHL the population is solely fresh water.

\subsubsection{Methodology}

The methodology of this system is based on two established facts. (1) The ions are in higher entropy than its solid crystal form. (2) The energy expenditure for ions separation is marginally small compare to energy generated by separated ions. This seem to be 
strange, but remember that ions have higher entropy than its associated solid crystal. Thus ions separation is analogous to activiation energy over a barrier to initiate chemical reaction.

To proof the technological feasibility, every system component should be carefully tested for its reliability in term of: efficiency, stability, perfomance and durability. Thus testing should follows a strict testing protocols suported by appropiate instrumentation and sensors, compulsory applied to all significant theoretical propositions of this system. Five crucial system components are first the VIWG capacitor which is design for hydrates destruction and ions migration, double layer plak investigation and electric curtain function to prevent back diffusion. The second crucial components is the graphite's terminals which acts as the site of electrons exchange with the ions influx and current moderator. The third component is power electronic circuitry, a.o is the DC/AC converter to 220 AC three phase and to high voltage (will be defined later) to connect to the existing National Electric Power Grid. The fourth components is the interfacing of sensors with the monitoring instruments including the mechatronics. The fifth components is the IT (information technology) which combined the monitoring system with data aquisition and data collection and for manual intervention if something goes wrong. Thus scientific validation has comparable significance with the engineering fabrication of the system.

\subsubsection{Instrumentation}

The above testing protocols should be supported by a number of very reliable component prototypes and instrumentations:

(1) VIWG (varnish insulated wire grid) capacitor.

(2) Different graphites rod and plate terminal for Auto-electrolyis test combined with VIWG. Note a plug and play technology should adopted for optimization of graphite collector size and configuration.

(3) DC/AC converter.

(4) The state of the art instrumentations and sensors: regulated DC power supply from 101000 DC volt; 2 four channel Oscilloscope; analog and digital multi meters, capacitance- meter, pH, salino, TDS; ASS (Atomic Adsorption Spectroscope); etc.

(5) Digital interfacing of sensors and measuring instruments trough USB connection.

(6) Data aquisition, monitoring and data-base storage through IT (information Technology).

(7) Manual intervention by operator through GUI (Graphical User interface).

The prototypes should be made from transparant strong materials such as acrylic.

\section{Result and Discussion}

The direct conversion of chemical seawater electrolytes produces theoretically $250 \mathrm{kJoule} / \mathrm{kg}$ standard seawater, which is only $0.55 \%$ of the fuel value of Texas's crude oil, U.S.A. Recall that the fuel value of crude oil and its associated hydrocracking technology is site specific in which the difference is due to impurities content. In our case the author has adopted the Texas's crude oil as benchmark.

The significant byproducts are 1 liter/s desalinated seawater (fresh water), Hydrogen and Chlorine gas, various rare metals dominated by Sodium metal. The conversion process is similar to fuel cell, with a 'priceless' unlimited availbility of seawater as feedstock. Furthermore this conversion process is very efficient and no pollution to the environment. Competitive advantages of this renewable technology is that the process is free from any kind of polution, high valued commercial by products, and the energy tariff can be as low as $30 \%$ of the current thermal generated electrical energy. Comparative advantages are unlimited supply of "priceless" seawater feedstock, scalable from $250 \mathrm{~kW}$ to $100 \mathrm{MW}$ plant, small foot print, absolutely no thermal polution and other polutions, power house for propulsion of any size and kind of ships.

A functional prototypes demonstration to facilitate fundamental research is absolutely neccessary to proof all the claims forwarded in this paper, follows by 100 MW Pilot Plant for industrial application before launching to the public. The neccesity of building fuctional prototypes is to make neccesary correction of the "ideal" model, forexample we have neglect the effect of random impuls forces attributed to thermal aggitation and gravity force. 


\subsection{Batch process for electrical desalination test}

This test validates the desalination theory by artifical electrical field. Recall that the field is created by VIWG capacitor. It is predicted that (1) after a few mcyc $=2.5$ ? $\mathrm{s}$ the population in the field will consists entirely of free water molecules. (2) The population of cations compartment are cations hydrates and free water molecules with basic $\mathrm{pH}$. The populations of anions compartment is anions hydrates with acid $\mathrm{pH}$. (3) No external additional energy is required for ions migrations, except the energy for VIWG charging. (4) Electric field prevent back diffusion of hydrates.

\subsection{Continuous process desalination test}

For continuos process, the system is equiped with a appropiate outlet, where the feedstock inflow is equal to the outfow. This test validates the tehcnological feasibility of continuous desalination process where the seawater feedstock is fed continuously into the system. In addition this test also (1) validates the existence of a thin EHL (Event Horizon Layer) and the MBIC (Multi Barrel Ions Cannon) and (2) the creation of SICC (Space Ions Clouds Capacitor) which creates high electric potential and current similar to the process atmospheric charged cloud. (3) No external additional energy is required for ions migrations, except the energy for VIWG charging. (4) The cations compartment will reach saturation where the energetic free ions influx is compensated by back diffusion and exite from the electric field through the outlet. (5) To prevent contamination of the already desalinated seawater, the REDOX reaction result should be continuously removed.

\subsection{Auto-electrolysis test}

The auto-electyrolysis is the site fuel cell like electrical energy generator, which is created by energetic collisions of ions with graphite electrodes where exchange of electrons occurs locally between ions and graphites surface. Thus the graphite's bar evolutes to becomes functionally as charge collector where the graphite's bar charge is equal to the ion'charge.

The REDOX occur locally at the collision point. The test covers two kind of test: (1) SICC Mode Test, where isolated ions compartment becomes a SICC. The electric charge are accumulated by ions as charge carrier and (2) Fuel Cell Mode Test, where the graphites collector are at the onset permanently connected

\section{Conclusion}

The artificial created electric field can be used for unlimited ions migration out of it, without needs of external energy once this field is established. The autoelectrolysis provide a fuel cell like process for electrical energy generation as long as the ions influx is maintained.

If the this ground breaking renewable energy reasearch is proven succesfully in terms of technology and economics through building a functional prototype where all aspects of design are optimized follows by $100 \mathrm{MW}$ fixed plant, than a new horizon of cheap and reliable energy generation based on chemical energy content of seawater electrolytes are on hand. Recall that $2 / 3$ of the the earth surface is covered by sea and therefore its feedstock guarantees unlimited supply. Since the system is multiscale a distributed isolated fixed plant is possible in particular in the geographical contex of Indonesia. Other important apllication is for propelling any kind of ship, where essentially the ship is moving in the unlimited supply of "chemical seawater energy".

\section{Acknowledgements}

This research is supported financially by MEDCO Energi Corporation since 2011.

\section{References}

Bogart Jr, T.F., Beasly, J.S. \& Rico G. (2004) Electronic Devices and Circuits. PEARSON,PRENTICE HALL.

Brown, LeMay, Burstein (1994) Chemistry. The Central Science, $10^{\text {th }}$ Ediditon. Prentice Hall.

Chang, R. (2007) Chemistry. McGraw Hill.

Dunn, P.F. (2005) Measurement and Data Analyisis for Engineering and Science. McGraw-Hill.

Fishbane, Gasiorowicz, \& Thorton (2005) PHYSICS for Scientist and Engineers with Modern Physics. Third Intern. Edition. Prentice Hall@.

Giancolli, D.C. (2005) PHYSICS. Principles and Apllications. Prentice Hall, $6^{\text {th }}$ Edition, Mc Graw Hill.

Hart, D.W. (2011). Power Electronics. McGraw Hill.

Jacob, J.M. (2002) Power Electronics: Principles \& Apllications. Delmar Inc. 
Johnson, C.D. (2006) Process Control Instrumentation Technology. $8^{\text {th }}$ Edition. Prentice Hall.

Kasap, S.O. (2006) Principles of Electronic Material and Devices. $3^{\text {rd }}$ Edition. Mc Graw Hill.

Rizzoni, G. (2003) Principles and Applications of Electrical Engineering. $4^{\text {th }}$ Edition. McGraw Hill.

Shames, I.H. (2003) Mechanics of Fluids. $4^{\text {th }}$ Edition. McGraw Hill.

Sumawiganda, S. (2011) Theory of Random Elastic Collisions between Solutes and Solvent due to thermal agitation. Propietary of CIETRIG.

Sumawiganda, S. (2012a) Sea Water Desalination Technology by strong Electric Field. Research Report to MEDCOEnergi, LAPIITB IndoWater Consulting.

Sumawiganda, S. (2012b) Auto-Electrolysis for Electrical Energy Generation. Research Report to MEDCOEnergi, LAPI-ITB IndoWater Consulting.

Sumawiganda, S. (2012c) Synchronization and load matching of freeions flux and Auto-Electrolysis for stable Electric Energy Conversion. Research Report to MEDCOEnergi, LAPI- ITB IndoWater Consulting.
Sumawiganda, S. (2012d) Thin Layer-Event-Horizon and Multi Barrel Ions Cannon. Site of an active ions migration in Electric Field Control Volume. CIETRIG, LAPI-ITB IndoWater Consulting.

Sumawiganda, S. (2013a) Note on Quantitative Characteristic of Space Capacitor Model. Propietary of CIETRIG.

Sumawiganda, S. (2013b) Short Note on Sodium-Chlorine Fuel Cell Cogeneration. Unpublished. Propietary of CIETRIG.

White, F.M. (2003) FLUID MECHANICS. $5^{\text {th }}$ Edition. McGraw Hill. 